\title{
Effects of Flavonoids from Scutellaria Baicalensis Georgi Stems and Leaves on Learning and Memory Ability of APP/PS1 Transgenic Mice
}

\author{
Ding Shengkai ${ }^{1}$, Miao Hong ${ }^{1}$, Gao Yang ${ }^{1}$, Guo Qing $^{2^{*}}$, Shang Yazhen ${ }^{1 *}$
}

${ }^{1}$ Institute of Traditional Chinese Medicine, Chengde Medical College / Hebei Province Key Research Office of Traditional Chinese Medicine Against Dementia / Hebei Province Key Laboratory of Traditional Chinese Medicine Research and Development / Hebei Key Laboratory of Nerve Injury and Repair. Anyuan Road, Chengde 067000, PR China.

${ }^{2}$ The Fourth Hospital of Shijiazhuang, Shijiazhuang, Hebei 050011, China

Citation: Dr. Shang Yazhen (2021) Effects of Flavonoids from Scutellaria Baicalensis Georgi Stems and Leaves on Learning and Memory Ability of APP/PS1 Transgenic Mice, Medical \& Clinical Research 6(2): 395-402.
${ }^{*}$ Corresponding authors

Dr. Shang Yazhen, Institute of Traditional Chinese Medicine, Chengde Medical College, Chengde 067000, PR China. Email: 973358769@qq.com Dr. Gao Qing, The Fourth Hospital of Shijiazhuang, Shijiazhuang, 050011, PR China

Submitted: 26 Jan 2021; Accepted: 01 Feb 2021; Published: 10 Feb 2021

\begin{abstract}
Introduction: APP/PS1 transgenic mice are established natively AD-like animal model. The present study used this animal model to study the effects of stems and leaves flavonoids from Scutellaria baicalensis Georgi (SSF) on APP/PS1 transgenic mice learning and memory ability with Morris water maze, jumping platform, dark box and shuttle box test. The results demonstrated that SSF markedly improve the learning and memory ability in APP/PS1 transgenic mice, and the four behavioral tests of Morris water maze, jumping platform, dark box and shuttle box are also suitable methods for evaluating the learning and memory ability of animals. Objective: To study the effects of SSF on the learning and memory ability in APP/PS1 transgenic mice by several behavioral experiment methods.
\end{abstract}

Methods: APP/PS1 transgenic mice were randomly divided into model group and three doses of SSF treated groups. While, the same period C57BL/6J mice were used as the control group. The APP/PS1 transgenic mice in the drug group were given continuous daily and orally administration of 50, 100 and $200 \mathrm{mg} / \mathrm{kg} \mathrm{SSF} \mathrm{for} 77$ days. On the day 51 of administration, four behavioral experimental methods, including Morris water maze, jumping platform, dark box and shuttle box were supplied to test the learning and memory ability of all mice and continually administered SSF during the test.

Results: Compared with the control group, Morris water maze test found that model group mice had a significant longer latency to find the hidden platform, shortened swimming time in the target quadrant, and reduced number of crossing platforms $(P<0.05, P<0.01)$. The detection of jumping platform and dark box showed that the latencies of the model group for jumping off the platform and entering the dark box were significantly shortened, and error times received by electric shocks was significantly increased $(P<0.05$, $P<0.01)$. The shuttle box test reported that the number of active avoidance in the model group was significantly decreased, while the times of passive avoidance and non-response were significantly increased $(P<0.05, P<$ 0.01). However, the three doses of SSF markedly shortened or prolonged the latency of APP/PS1 transgenic mice to find the hidden platform, jump off the platform and enter the dark box, as well as reduce the errors times of electric shock $(P<0.05, P<0.01)$.

Conclusion: SSF can improve the learning and memory impairment of APP/PS1 mice by different behavioral test.

Keywords: Flavonoids from Scutellaria baicalensis Georgi Stems and Leaves; APP/PS1 Transgenic Mice; Morris Water Maze; Jumping Platform; Dark Box; Shuttle Box; Learning and Memory Ability 


\section{Introduction}

Alzheimer's disease (AD) is a global public health crisis [1]. According to the data of "World Alzheimer Report 2019 Attitudes to Dementia" released by the Alzheimer's disease International (ADI), there are more than 50 million AD patients worldwide in 2019 and will reach 152 million by 2050 . The report also estimated that the current global treatment cost will be 1 trillion $\$$ and will enhance to 2 trillion dollor by 2030, which will have a heavy economic and psychological burden to the families and society [2].

$\mathrm{AD}$ is a degenerative disease of the central nervous system, and the patients with progressive learning and memory impairment and cognitive dysfunction are the main clinical symptoms [3]. A variety of animal models were established in the laboratory to study the pathophysiology and drug screening of AD. Among them, the transgenic animal model of AD was based on genetic theory. Wengenack et al. used gene targeting technology to breed APP/ PS1 double transgenic mice, which showed typical neurology and dysfunction like AD, namely characteristic neuronal pathological changes and learning and memory impairment. The model of APP/ PS1 transgenic mice is the closest to the clinical manifestations and neuropathological changes of AD patients, and is also usually used to study the pathophysiology and new drug research in the laboratory for $\mathrm{AD}[4,5]$.

SSF is extracted from the stems and leaves of Scutellaria baicalensis Georgi and scutellarin is the main ingredient. Studies have shown that SSF not only has the effects in antibacterial, antiviral, anti-inf lammatory, antioxidant, hypolipidemic and myocardial protection, but also can improve the learning and memory function in many kinds of AD model animals [6-8]. However, it has not been reported whether SSF can improve the learning and memory impairment of mice with transgenic mice. The aim of present study is to investigate the effects of SSF on the learning and memory ability for APP/PS1 transgenic mice.

\section{Methods and Materials}

\section{Experimental Animal}

APP/PS1 and C57BL/6J mice were purchased from Beijing HFK Bioscience Co., Ltd., experimental animal production license No.: SCXK (Beijing) 2019-008. They were raised in the barrier environment of the Experimental Animal Center of Chengde Medical College (license No.: SYXK (Ji) 2117-001). The temperature was maintained at $23-25^{\circ} \mathrm{C}$, the humidity was $55 \% \pm 5 \%$, and the light/dark cycle of $12 \mathrm{~h} / 12 \mathrm{~h}$ was maintained, and the mice were allowed to eat freely and adapt to the experiment after 1 week purchased. The animal experiment process followed the relevant ethical requirements of the "Guiding Opinions on being kind to Experimental Animals" issued by the Ministry of Science and Technology of the People's Republic of China in 2006, and has been approved by the Ethics Committee of Chengde Medical College (license No.: CDMULAC-20190226-002).

\section{Drugs and Testing Equipment}

Scutellaria baicalensis Georgi was identified by Professor Zhao Chunying, Chengde Medical College and Scutellaria baicalensis Georgi stems and leaves flavonoids were prepared by the Institute of Traditional Chinese Medicine, Chengde Medical College (Lot.: 20100618). Morris water maze (Institute of Medicine, Chinese
Academy of Medical Sciences), SuperSdt jump platform, Super APAS shuttle box and SuperPas avoidance dark box (Shanghai Xinruan Information Technology Co., Ltd.).

\section{Experimental Design}

30 male 4-month-old APP/PS1 transgenic mice were randomly divided into four groups with 6 mice in each group, including model group and three doses of SSF-treated groups. 6 C57BL/6J mice served as the control group. The three doses of SSF-treated groups were orally daily administrated SSF at dose of 50,100 and $200 \mathrm{mg} / \mathrm{kg}$ for 77 days. The control group and APP/PS1 model group were given the same volume of saline, respectively, for 77 days.

On the day 51 of administration, the Morris water maze was used to test the learning and memory abilities of the mice for 11 consecutive days. On the day 64 of administration, the passive avoidance memory acquisition ability of the mice was tested by jumping platform for 2 consecutive days. On the day 68 of administration, the dark box was used to detect the non-spatial passive avoidance memory ability of the mice for 2 consecutive days. On the day 72 of administration, the non-statement memory ability of mice was tested by shuttle box for 5 days. After the end of each behavioral test, the mice rested for 2 days and began a new round of testing.

Behavioral Experimental Methods to Detect the Learning and Memory Ability of Mice Morris Water Maze Equipment The Morris water maze is a circular pool with a diameter of 120 $\mathrm{cm}$ and a depth of $50 \mathrm{~cm}$, with a water depth of $31.5 \mathrm{~cm}$ and temperature at $23 \pm 2{ }^{\circ} \mathrm{C}$ [9]. The water was become white by adding proper amount of edible white colorant. The pool is imagined equally divided into four quadrants, and mark four distinctive markers at equal distances around the pool wall as water entry points. According to the experimental requirements, a cylindrical transparent plexiglass platform with a diameter of $10 \mathrm{~cm}$ and a height of $30 \mathrm{~cm}$ was placed in the middle of one of the quadrants, hidden under the water surface for $1.5 \mathrm{~cm}$. A camera is installed above the pool, which is connected to the graphic analysis software of the acquisition card to link the computer for capturing the swimming behavior of the mice (measured by latency, swimming trajectory and crossing times and other behavioral targets.). The latency of mouse was recorded when they found the hidden platform. When the mouse found and climb the platform within 60 $\mathrm{s}$, they were allowed to stay there for $20 \mathrm{~s}$. If a mouse did not find the platform within $60 \mathrm{~s}$, the experimenter guided the mice to climb onto the platform and stayed there for $20 \mathrm{~s}$, and the latency was recorded as $60 \mathrm{~s}$. The measurement of mice learning and memory abilities were conducted twice a day in the morning and afternoon, in which trial one point was far away from the platform and one point was close to the platform. The average of the 4 latencies was used as the performance of the day. During the test in the Morris water maze, all the surrounding spatial signals were guaranteed to be constant.

\section{Test of Memory Acquisition Ability of Mice}

The positioning navigation trial was conducted to test the memory acquisition ability of mice on the day 1-5 of Morris water maze task, corresponding to the day 51-55 of administration. The hidden 
platform was placed in the second quadrant, and the average latency of the test at 4 points per day was taken as the day's score, and SSF continued during the test.

\section{Test of Memory Retention Ability of Mice}

On the day 6 of the probe trial, corresponding to the day 56 of administration, the memory retention ability of the mice was measured. The hidden platform was removed from the water maze, and the swimming trajectory of the mice in the water maze within $60 \mathrm{~s}$ and the swimming time and number of crossings in the target quadrant were recorded. SSF was continually administered during the test.

\section{Test of Memory Re-Learning Ability of Mice}

On the day 7-10 of Morris water maze task, corresponding to the day 57-60 of administration, the opposite platform trial was performed to detect the re-learning ability of mice. The hidden platform in the water maze was placed the opposite side of the original position, and the test was continued for 4 days. The SSF was continued during the test.

\section{Test of Swimming Speed of Mice}

In order to exclude the difference in swimming speed caused by individual differences of mice, the swimming speed of the mice was measured on the day 11 of the Morris water maze, corresponding to the day 61 of administration. The platform was over the $1.5 \mathrm{~cm}$ of the water surface. The swimming distance and time of the mice for reaching the visible platform was being used to evaluate the swimming speed of mice.

\section{Jumping Platform Experiment}

In the open space, animals spend most of their time moving around the edges and corners to test the passive avoidance memory acquisition ability animals, and the jumping platform box is equipment for detecting passive avoidance memory acquisition ability of the animal. The jumping platform box is designed with an insulated cylindrical platform in the center in the square space, and a copper grid is placed at the bottom and can be electrified. When the animal is placed on the platform, the animal will almost immediately jump off the platform and explore the surroundings according to their nature. When the animal jumped off the platform the animal was electric shock, and then, the animal jump on the platform again to avoid the electric shock. Sometime, some animals will jump to the energized copper grid again, and then quickly jump back to the platform after being the electric shock, repeating the cycle [10].

Before the training, mice were put in the jumping platform box to familiar to the environment for $3 \mathrm{~min}$. When the experiment was conducted, the copper grid was electrified by $32 \mathrm{~V}$ alternating current and the mice were trained for $5 \mathrm{~min}$. The first time of each mouse jumping off the platform and the number of electric shock of mouse jumped off the platform were recorded within $5 \mathrm{~min}$ of the training period. This time and number of electric shock is named the latency and the number error, respectively. If a mouse stayed on the platform for more than $5 \mathrm{~min}$, the latency was recorded as $300 \mathrm{~s}$ and to be the score of mouse learning test. The above experiments were repeated $24 \mathrm{~h}$ later, as memory test results. The jumping platform box needs to be wiped with $75 \%$ ethanol before the next mouse test was started, in order to remove the odor of the previous mouse.

\section{Dark Box Experiment}

The dark box was used mice the learning and memory ability of non-spatial passive avoidance, according to the mice life habit for liking dark and avoiding light [10]. The dark box is composed of two parts of the same size as the light box and the dark box. A semi-circular small hole is at the middle baffle of the two boxes for mice to pass through. There are copper grids that can be energized at the bottom, and the electrical stimulation is placed at dark box. When the mice enter the dark box, the mice will be electrically shocked.

In the test, the mice were placed in the bright box by their back to the middle baffle hole. When the mice entered the dark box, and then immediately escape to the bright box because of electrical shock. The process was performed after $24 \mathrm{~h}$ for testing the mice memory. The first time of each mouse entering the bright box and the number of electric shock of mouse were recorded within 3 min of the test. This time and number of electric shock is named the latency and the number error, respectively. If a mouse did not enter the dark box within 3 min, the dark avoidance latency was recorded 180s. The dark box needs to be wiped with $75 \%$ ethanol before the next mouse test was started, in order to remove the odor of the previous mouse.

\section{Shuttle Box Experiment}

The active avoidance learning is a basic behavioral phenomenon, which reflects the animal's ability of non-statement memory [11]. The shuttle box is usually used to test the non-statement memory of animals. The shuttle box is consisted of non-toxic, light-proof and sound-proof medical ABS plastic and with two identical compartments [12]. There is semi-circular small hole at the bottom for animals passing through the two compartments. A copper grid is put at the bottom for the electrical stimulation. A LED bulb and a loudspeaker set the top of the box provide the light stimulation and sound stimulation, respectively. A camera placed on the top of the box is linked to the data collected system, which tracks and analyzes the animals' behavior in the shuttle box. The mice were put into the shuttle box $5 \mathrm{~min}$ for acclimatizing the box environment ahead of the training. On the beginning of the experiment, the experiment parameters need to be set, including conditioned stimulation of buzzing sound and light for $5 \mathrm{~s}$, and unconditioned stimulation of electrical shock by $5 \mathrm{~s}, 0.5 \mathrm{~mA}$.

In the induction training phase, the mice were put into the left box by facing the wall. The test stated after the mice acclimate the environment the box for $30 \mathrm{~s}$. After the mice received the conditioned or unconditioned stimulation, the mice escaped the stimulation to the opposite side from the round hole. The contralateral side was also given the same conditioned and unconditioned stimulation by an interval of $30 \mathrm{~s}$. The same process was repeatedly and kept 300 s.If the mouse did not escape the both conditioned and unconditioned stimulation, the record is as no response. This training was lasted for $4 \mathrm{~d}$. The test was carried out on the $5 \mathrm{~d}$ trained and the number of active avoidance, passive avoidance and non-response were recorded as the mice's learning and memory score. The shuttle box needs to be wiped with $75 \%$ 
ethanol before the next mouse test was started, in order to remove the odor of the previous mouse.

\section{Statistical Analysis}

All data are analyzed by SPSS21.0 Statistical Software, and the values are expressed as Mean $\pm \mathrm{SEM}$. Morris water maze data was processed by two-way ANOVA. The data of jumping platform, shuttle box and dark box are processed by one-way ANOVA. $\mathrm{P}<0.05$ was considered statistically significant.

\section{Results}

Morris Water Maze Test the Effect of SSF on Learning and Memory of APP/PS1 Mice

The Effect of SSF on APP/PS1 Mice Memory Acquisition Ability by Morris Water Maze Test

The positioning navigation trial on day1-5in the Morris water maze was used to measure the memory acquisition ability of mice. As shown in Figure 1, the time for mice in the model group to find the hidden platform was significantly increased $(\mathrm{P}<0.01)$. On the day 1-3 of the test, there was no statistical significance between the control group and model group ( $\mathrm{P}>0.05)$. But on the day $4-5 \mathrm{~d}$ test, the model group spent $50.8 \%$ and $58.12 \%$ higher time to find the hidden platform than that of control group $(\mathrm{P}<0.01)$. Interesting, On the day 4-5 d of water maze test, the latency of mice decreased by $29.26 \%(\mathrm{~d} 4, \mathrm{P}<0.01)$ and $47.01 \%(\mathrm{~d}, \mathrm{P}<0.01)$ in SSF $50 \mathrm{mg} /$ $\mathrm{kg}$ group, by $58.67 \%(\mathrm{~d} 4, \mathrm{P}<0.01)$ and $57.26 \%(\mathrm{~d} 5, \mathrm{P}<0.01)$ in $\mathrm{SSF} 100 \mathrm{mg} / \mathrm{kg}$ group, and by $76.65 \%(\mathrm{~d} \mathrm{4}, \mathrm{P}<0.01)$ and $75.12 \%$ (d 5, P<0.01) in SSF $200 \mathrm{mg} / \mathrm{kg}$ group, respectively.

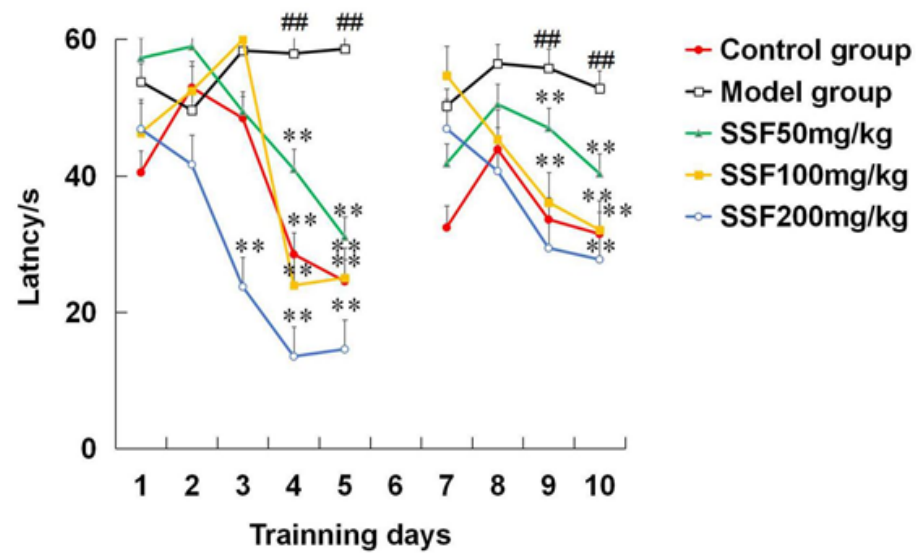

Figure 1: Effects of SSF on memory acquisition and re-learning impairment in APP/PS1 mice. The positioning navigation trial was used to evaluate memory acquisition by 5 consecutive days on the day 1-5 in the Morris water maze test. The reversal trial was used to evaluate memory re-learning of rats by 4 consecutive days on day 7-10 in the Morris water maze test. The line graph plots showed the mean latency to find the hidden platform for each group on the day 1-5 and 7-10 in the Morris water maze test. Data were analyzed by two-way ANOVA (day $\times$ group) with repeated measures. Mean \pm SEM. ${ }^{\#} \mathrm{P}<0.01$, compared with control group. $* * \mathrm{P}<0.01$, compared with model group.

The Effect of SSF on APP/PS1 Mice Memory Retention Ability by Morris Water Maze Test

On the day 6 in the Morris water maze test, the probe trial was used to detect the memory retention ability of mice. The result in Figure 2 showed that the swimming time, platform crossing times (Figure 2A) and swimming trajectory (Figure 2B) in the target quadrant were significantly decreased in the model group mice. The swimming time and the number of crossing platforms in the target quadrant of model group reduced $32.61 \%(\mathrm{P}<0.01)$ and $63.24 \%$ ( $\mathrm{P}<0.01)$, as compared with control group. however, APP/ PS1 transgenic mice, three doses of SSF increased the swimming time and platform crossing times of APP/PS1 transgenic mice in the target quadrant by $11.58 \%, 55.67 \%(50 \mathrm{mg} / \mathrm{kg}, \mathrm{P}<0.05$, $\mathrm{P}<0.01), 43.06 \%, 51.55 \%$ (100 mg/kg, $\mathrm{P}<0.01), 26.33 \%, 59.94 \%$ $(200 \mathrm{mg} / \mathrm{kg}, \mathrm{P}<0.01)$, respectively.
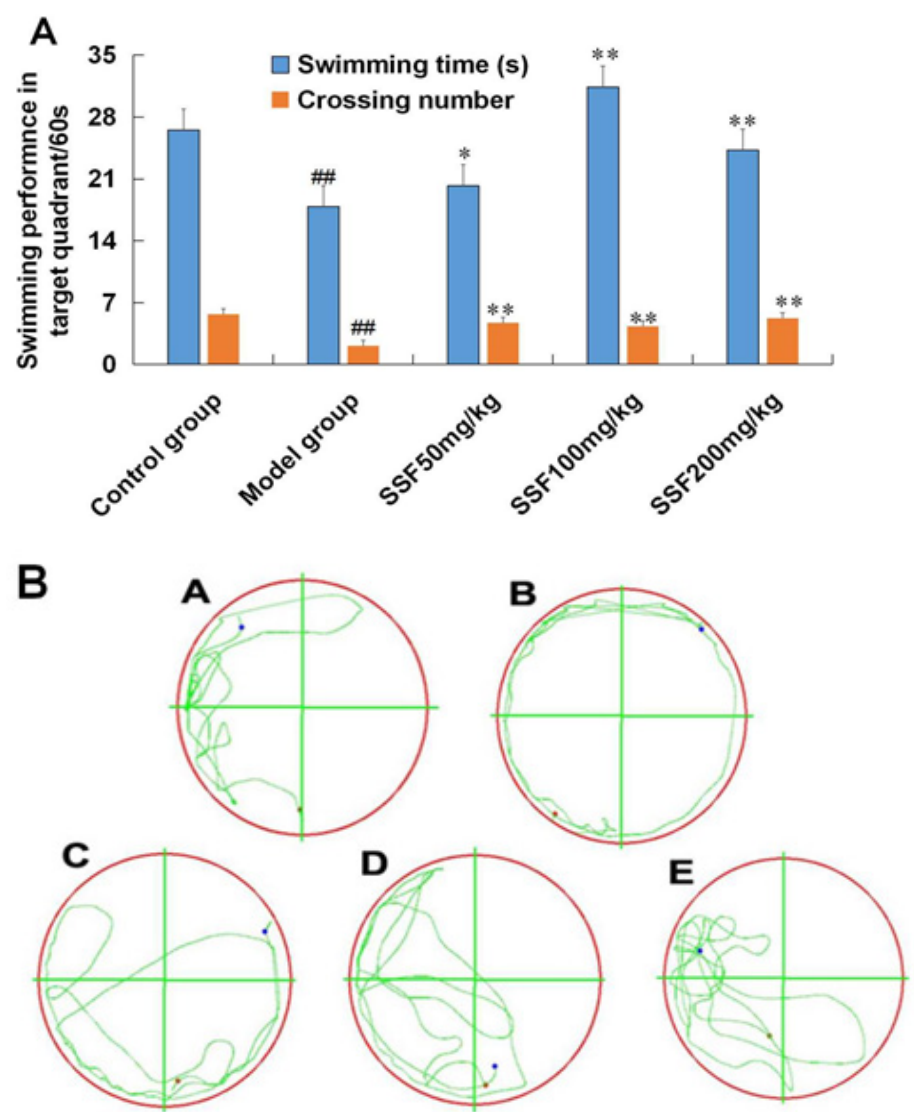

Figure 2: Effects of SSF on memory retention in APP/PS1 mice. The probe trial was used to evaluate memory retention of mouse on day 6 in the Morris water maze test. A: Mean of swimming time, and crossing number, respectively, in the target quadrant within $60 \mathrm{~s}$ in the probe trial (no platform). Data were analyzed by one-way ANOVA with the multiple-range test. Mean $\pm \mathrm{SEM}$, ${ }^{\#} \mathrm{P}<0.01$, compared with control group. ${ }^{*} \mathrm{P}<0.05$, $* * \mathrm{P}<0.01$, compared with model group. B: Typical swimming-tracking paths of mice in probe trial. A: Control group, B: model group, C: SSF $50 \mathrm{mg} / \mathrm{kg}$ group, D: SSF $100 \mathrm{mg} / \mathrm{kg}$ group, E: SSF 200 $\mathrm{mg} / \mathrm{kg}$ group.

The Effect of SSF on APP/PS1 Mice Memory Re-learning Ability by Morris Water Maze Test

The opposite platform trial on day 7-10 in the Morris water maze was used to assay the re-learning ability of mice. As shown in Figure 1, the model group took longer latency to find the hidden 
platform than control group. The latency of model group increased $39.76 \%$ (d 9, $\mathrm{P}<0.01$ ) and 40.40\% (d 10, $\mathrm{P}<0.01$ ), respectively, as compared with control. However, the mice in the three dose of SSF groups spend shorter time to find the platform in 7-10 d test, but no statistical significance $(\mathrm{P}>0.05)$. In the Morris water maze 9-10 days test, compared with model group, the latency decreased by $15.7 \%(\mathrm{~d} 9, \mathrm{P}<0.05)$ and $23.65 \%(\mathrm{~d} 10, \mathrm{P}<0.01)$ in SSF $50 \mathrm{mg} /$ $\mathrm{kg}$ group, decreased by $35.25 \%(\mathrm{~d} 9, \mathrm{P}<0.01)$ and $39.83 \%(\mathrm{~d} 10$, $\mathrm{P}<0.01)$ in SSF $100 \mathrm{mg} / \mathrm{kg}$ group, and decreased by $47.72 \%(\mathrm{~d}$ $9, \mathrm{P}<0.01)$ and $47.48 \%$ (d 10, $\mathrm{P}<0.01)$ in $\mathrm{SSF} 200 \mathrm{mg} / \mathrm{kg}$ group, respectively.

The Effect of SSF on APP/PS1 Mice Swimming Speed by Morris Water Maze Test

The swimming speed of mice was measured by the visual platform test on the day 11 of the Morris water maze. There was no significant difference in the time required for each group of mice to find the visual platform $(\mathrm{P}>0.05)$. This result showed that there is no obvious difference in swimming physical strength of all group mice, which indicated that individual differences was excluded for disturbing the results of Morris water maze detection.

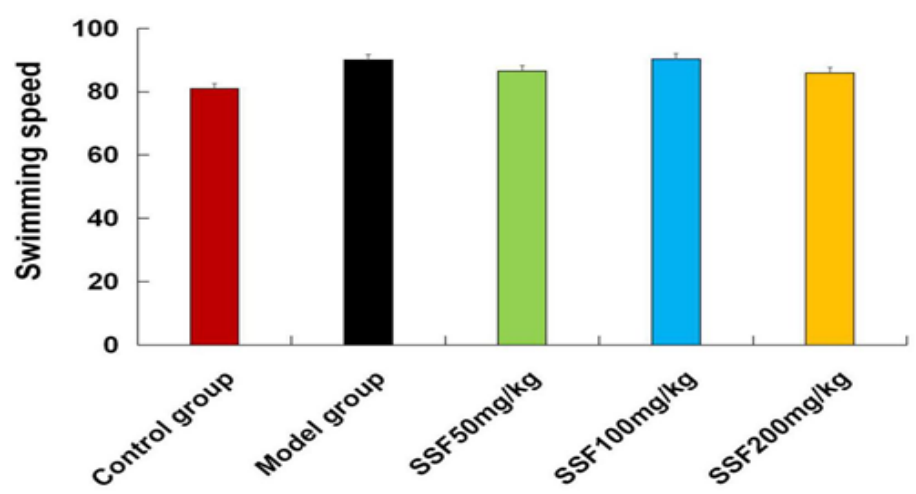

Figure 3: Effect of SSF on swimming speed by in APP/PS1 mice. The mice rat swimming speed was calculated by the visible platform trial on the day 11 of the Morris water maze test. Data were analyzed by one-way ANOVA with the multiple-range test. Mean \pm SEM. $\mathrm{n}=6$.

The Effect of SSF on APP/PS1 Mice Short-term Passive Avoidance Memory Acquisition Ability by Jumping Platform Test

The second day of jumping platform test was used to assay the mice the short-term memory acquisition ability. As shown in figure 4 , the latency was reduced by $57.95 \%(\mathrm{P}<0.01)$ and the errors times increased by $84.21 \%(\mathrm{P}<0.01)$ in model group, as compared with the control group. Three doses of SSF increased the latency $51.68 \%$ by SSF $50 \mathrm{mg} / \mathrm{kg}(\mathrm{P}<0.01), 52.08 \%$ by $\mathrm{SSF} 100 \mathrm{mg} / \mathrm{kg}$ $(\mathrm{P}<0.01)$ and $54.30 \%$ by SSF $200 \mathrm{mg} / \mathrm{kg}(\mathrm{P}<0.01)$, and the number of errors decreased by $50.95 \%(\mathrm{SSF} 50 \mathrm{mg} / \mathrm{kg}, \mathrm{P}<0.01), 68.42 \%$
$(\mathrm{SSF} 100 \mathrm{mg} / \mathrm{kg}, \mathrm{P}<0.01)$ and $71.93 \%(\mathrm{SSF} 200 \mathrm{mg} / \mathrm{kg}, \mathrm{P}<0.01)$, respectively, higher than that of control group.
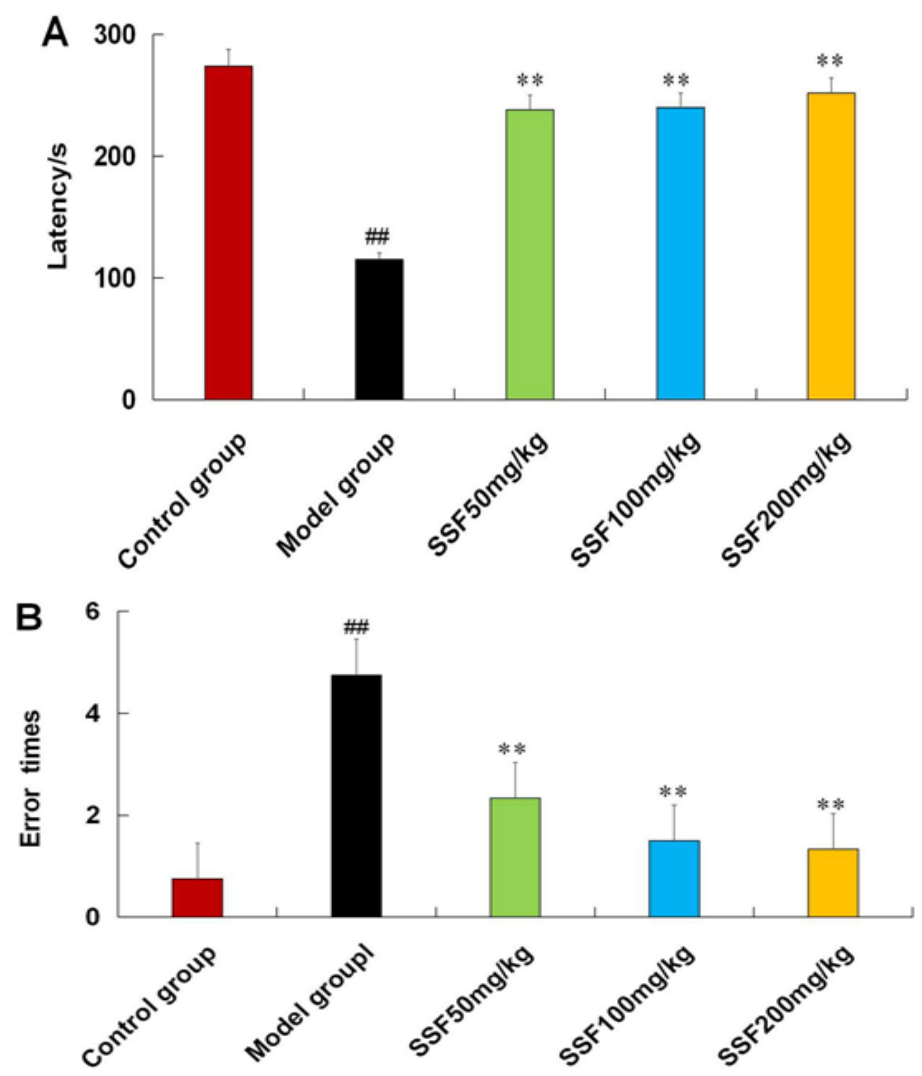

Figure 4. The effect of SSF on short-term memory acquisition ability in APP/PS1 mice was test by jumping platform. A: The latency of jumping platform during the test. B: Errors times jumping platform during the test. Data were analyzed by one-way ANOVA with the multiple range test. Mean $\pm \mathrm{SEM},{ }^{\#} \mathrm{P}<0.01$, compared with control group. $* * \mathrm{P}<0.01$, compared with model group.

The Effect of SSF on APP/PS1 Mice Memory of Non-spatial Passive Avoidance Ability by Dark Box Test

The second day of dark box test was used to determine the memory of non-spatial passive avoidance. Figure 5 showed that the latency of reduced by $69.04 \%(\mathrm{P}<0.01)$, and the errors times increased by $65.79 \%(\mathrm{P}<0.01)$ in model group, as compared with control group. However, three doses of SSF increased the latency by $59.99 \%$ (SSF $50 \mathrm{mg} / \mathrm{kg}, \mathrm{P}<0.01$ ), 63.08\% (SSF $100 \mathrm{mg} / \mathrm{kg}, \mathrm{P}<0.01$ ), 61.78\% (SSF $200 \mathrm{mg} / \mathrm{kg}, \mathrm{P}<0.01)$, respectively. The errors times decreased by $70.01 \%(\mathrm{SSF} 50 \mathrm{mg} / \mathrm{kg}, \mathrm{P}<0.01), 56.52 \%$ (SSF $100 \mathrm{mg} / \mathrm{kg}$, $\mathrm{P}<0.05$ ) and $59.42 \%$ (SSF $200 \mathrm{mg} / \mathrm{kg}, \mathrm{P}<0.05)$, respectively. 

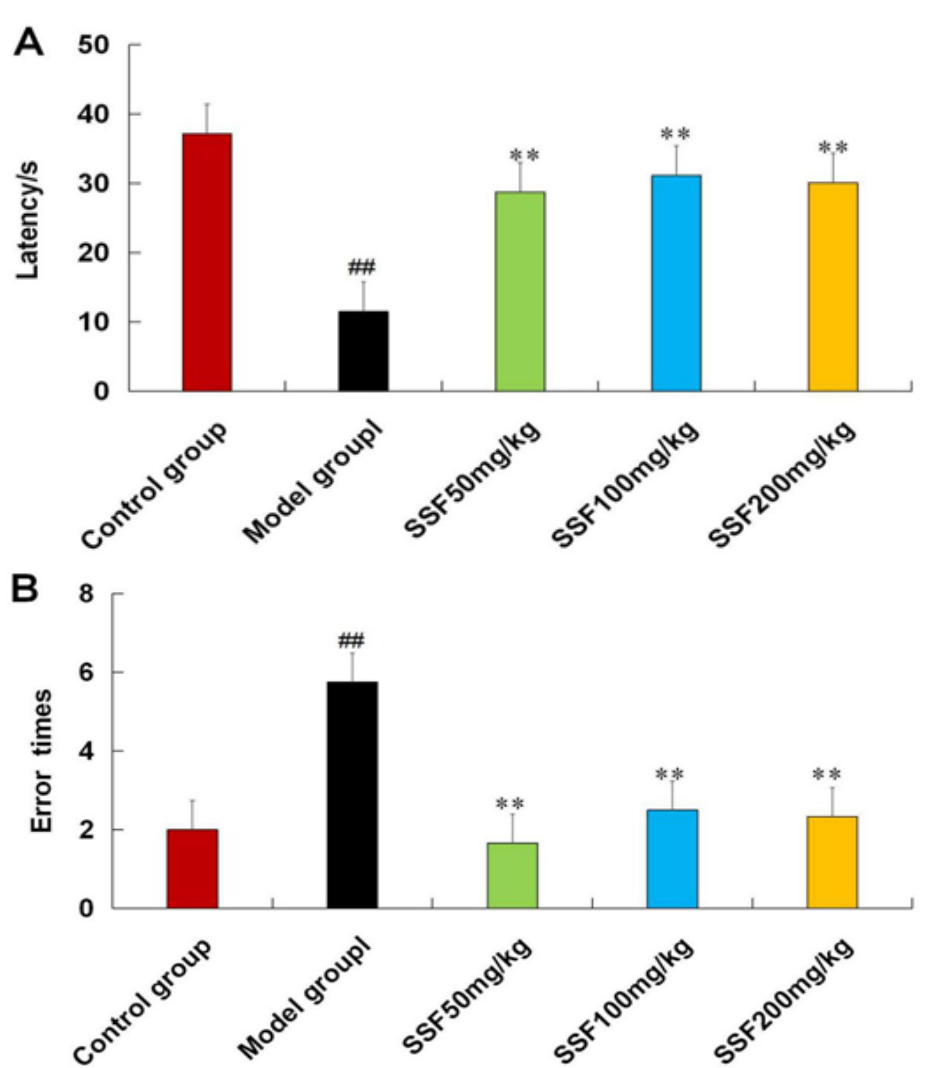

Figure 5: The effect of SSF on non-spatial passive avoidance in APP/PS1 mice was test by dark box. A: The latency of dark box during the test; B: Errors times of dark box during the test. Data were analyzed by one-way ANOVA with the multiple-range test. Mean $\pm \mathrm{SEM},{ }^{\#} \mathrm{P}<0.01$, compared with control group. ${ }^{* *} \mathrm{P}<0.01$, compared with model group.

The Effect of SSF on APP/PS1 Mice Non-statement Memory Ability by Shuttle Box Test

The non-statement memory ability of mice was detected using shuttle box by the number of active avoidance, passive avoidance and non-reaction of mice. The results shown in figure 6 , compared with the control group, the active avoidance times decreased by $49.28 \%(\mathrm{P}<0.01)$, the passive avoidance times increased by $72.5 \%$ $(\mathrm{P}<0.01)$, and the non-reaction times increased by $91.1 \%(\mathrm{P}<0.01)$, respectively in model group. It is interest that three doses of SSF increased by $53.95 \%$ (SSF $50 \mathrm{mg} / \mathrm{kg}, \mathrm{P}<0.01$ ) , 48.53\% (SSF 100 $\mathrm{mg} / \mathrm{kg}, \mathrm{P}<0.01$ ) and $53.95 \%$ (SSF $200 \mathrm{mg} / \mathrm{kg}, \mathrm{P}<0.01$ ) in the active avoidance number; reduced by $90 \%$ (SSF $50 \mathrm{mg} / \mathrm{kg}, \mathrm{P}<0.01$ ), $70 \%$ (SSF $100 \mathrm{mg} / \mathrm{kg}, \mathrm{P}<0.01$ ), and 90\% (SSF $200 \mathrm{mg} / \mathrm{kg}, \mathrm{P}<0.01$ ) in the passive avoidance times, reduced by $91.11 \%$ (SSF $50 \mathrm{mg} / \mathrm{kg}$, $\mathrm{P}<0.01$ ), 73.3\% (SSF $100 \mathrm{mg} / \mathrm{kg}, \mathrm{P}<0.01$ ) and 77.33\% (SSF 200 $\mathrm{mg} / \mathrm{kg}, \mathrm{P}<0.01$ )in the non-response number respectively.

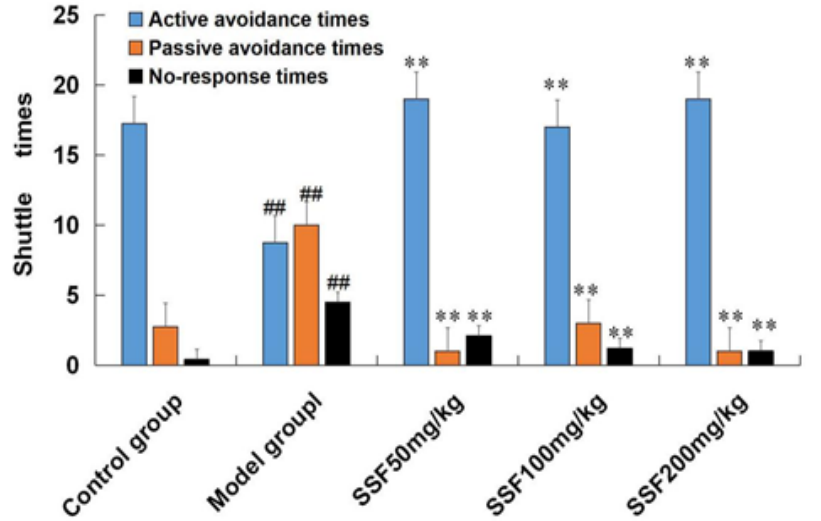

Figure 6: Effects of SSF on non-statement memory in APP/ PS1 mice was test by shuttle box. Active avoidance times, passive avoidance times and non-reaction times were measured, respectively. Data were analyzed by one-way ANOVA with the multiple-range test. Mean $\pm \mathrm{SEM},{ }^{\#} \mathrm{P}<0.01$, compared with control group. ${ }^{* *} \mathrm{P}<0.01$, compared with model group.

\section{Discussion}

Experimental evaluation of animal behavior is widely used in neuroscience fields, especially in the evaluation of cognitive dysfunction of animal models and the physiological mechanisms of the related diseases [13,14].The learning and memory impairment is the most important clinical manifestation in $\mathrm{AD}$, and the behavioral monitored is the most common method to assess learning and memory function [15]. In the laboratory, there are many strategies to detect animal behavior including the Morris water maze, the Barnes maze and the eight-arm maze, and other methods like shuttle box, jumping platform and dark box.

The Morris water maze was proposed and designed by British neurologist Morris in 1981. It is used to test the hippocampusdependent learning and memory abilities of animals [16], and is regarded as the "gold standard" in behavioral neuroscience research [17]. Then, the Morris water maze test has become the first classical method for evaluating the spatial learning and memory in laboratory, especially in the study of various cognitive disorders, such as AD [18]. The Morris water maze mainly can test the learning and memory of three phases, including the memory acquisition, memory retention and memory re-learning. In the Morris water maze test, the positioning navigation trial is used to measure the animal memory acquisition. This tested principle is based on that the animal is familiar to the spatial location of the surrounding subject through repeated learning and takes the shorter latency to find the hidden platform. The latency will be prolonged, when the spatial memory ability decreases of animal. The probe trial is used to detect the animal memory retention. This tested principle is based on that the animal memorizes the spatial 
position of the platform after the learning. The swimming time and the crossing number of animal in the target quadrant express the animal memory retention ability in the probe trial. The opposite platform trial is used to detect the memory re-learning ability of animals. This tested principle is based on that the brain extracts something from previous knowledge and experience [14,19].

The passive avoidance experiment is that the animal avoids certain disgusting things by learning to get rid of certain behaviors. The tested methods include the jumping platform, the dark box and upward avoidance experiment and other avoidance tests. Both the jumping platform and black box can all detect the passive memory acquisition ability through avoiding the electric shock [15]. The jumping platform experiment makes use of the living habits of animals in the open space, most of the time at the edges and corners, there mainly tests the animal's short-term memory acquisition ability, which not only observes the influence of drugs on the memory process, but also observes the influence in learning ability. This test is regarded the high sensitivity. The dark box test is designed according to the mice's the dark habits, which belongs to passive avoidance response, and mainly represents the nonspatial memory of animals. It is highly sensitive to the memory process, especially to the re-learning memory [20]. The learning and memory ability of the animal was tested by the latency of jumping off the platform and entering the dark box or the number of errors by electric shocked.

The shuttle box test is used to test the non-statement memory ability. Non-statement memory refers to the memory that needs repeated training and accumulated through long-term repeated experience, which is related to actual operation and practice. The mice can actively avoid harmful stimulation and non-statement memory acquired through shuttle training [11]. In the test, the light or sound and electrical stimulation were used to detect the shuttle behavior, including active avoidance number, passive avoidance number and no response number. When the number of active avoidance was decreased, and the number of electric shock was increased, the learning and memory ability of animal was indicated poor.

In the present study, Morris water maze, jumping platform, dark box and shuttle box were used to study the effect of SSF on the learning and memory ability in APP/PS1 transgenic mice. The results showed that the latency of APP/PS1 transgenic mice to find the hidden platform was significantly prolonged, the swimming time in the target quadrant was significantly shortened, and the number of times crossing the platform was significantly reduced in the Morris water maze test, compared with the control group. In the detection of jumping platform and dark box, the latency of APP/PS1 transgenic mice jumping off the platform and entering the dark box was significantly shortened, and errors times received by electric shocks was significantly increased. In the shuttle box test, APP/PS1 transgenic mice was found that the active avoidance times was significantly decreased, while the passive avoidance times and non-response times were significantly increased. However, three doses of SSF markedly shortened or prolonged the latency of APP/PS1 transgenic mice in varying degrees and reduced errors times by electric shock. These results indicated that SSF administered for 77 days can significantly improve the learning and memory ability of APP/PS1 transgenic mice, and the four behavioral tests of Morris water maze, jumping platform, dark box and shuttle box are also suitable methods to detect the learning and memory ability of animals.

\section{Conclusion}

$\mathrm{AD}$ is a progressive and irreversible degenerative disease of the central nervous system in the middle-aged and elderly population. The clinical manifestations of AD patients are the gradual decline of memory ability and the impairment of learning and cognitive function, and even damage their independent living ability. The present study used APP/PS1 transgenic mice, natively AD-like model, to detect their the memory acquisition, memory retention, memory re-learning, passive avoidant learning and memory and non-statement memory by Morris water maze, jumping platform, dark box and shuttle box. The results showed that SSF was found to have an improvement in the learning and memory impairment of APP/PS1 transgenic mice, which suggested that SSF may be beneficial effect in treatment of AD. Morris water maze, jumping platform, dark box and shuttle box are also suitable methods for evaluating the learning and memory ability of animals.

\section{Human and Animal Rights}

No humans were used. All reported experiments on animals were followed in The guidelines for animal were in accordance with the Regulations of Guiding Opinions on being kind to Experimental Animals issued by the Ministry of Science and Technology of the People's Republic of China on Sep. 30, 2006.

\section{Acknowledgement}

The project was supported by Hebei Provincial Natural Science Foundation (No. C2009001007, H2014406048, H2019406063); Hebei Provincial Administration of Traditional Chinese Medicine (No. 05027, 2014062); Hebei Provincial Education Department (No. ZD20131022, ZD2019057); Hebei Provincial Hundred Outstanding Innovated Talents (First Batch); Key Subject Construction Project of Hebei Provincial College; Technology Innovation Guidance Project-Science and Technology Work Conference of Hebei Provincial Department of Science and Technology; The Key Development Subject of Pharmacology of Traditional Chinese Medicine of Chengde Medical College (No.[2020]49) and Science and Technology Innovation Team Construction Project of Chengde Medical College of China. (No. [2020]50) for financial.

\section{Conflict of Interest}

The authors declare no conflict of interest, financial or otherwise.

\section{Reference}

1. Prince M, Ali GC, Guerchet M, Prina AM, Albanese E, et al. (2016) Recent global trends in the prevalence and incidence of dementia, and survival with dementia. Alzheimers Res Ther 8: 23-26.

2. Alzheimer's Disease International (ADI) (2019) World Alzheimer Report 2019: attitudes to dementia. London: ADI 2019: 1-160.

3. Zhang LL, Song WS, Wang K, Sun WM, Wang YF (2017) Progress in mechanism of pathogenesis and Medical Treatment of Alzheimer's disease. World Chin Med 12:1200-1208. 
4. Lewis J, Dickson DW, Lin WL, Chisholm L, Corral A, et al. (2001) Enhanced neurofibrillary degeneration in transgenic mice expressing mutant tau and APP. Science 293:1487-1491.

5. Li YL (2013) Establishment of experimental models for Alzheimer's disease research. Int J Neurosci 123:823-831.

6. Xi YL, Shang YZ (2007) Research progress on the stems and leaves of Scutellaria baicalensis Georgi. J Chengde Med Col 24: 293-295.

7. Shang YZ, Gong MY, Zhou XX, Li ST, Wang BY (2001) Improving effects of SSF on memory deficits and pathological changes of neural and immunological systems in senescent mice. Acta pharmacol Sin 22:1078-1083.

8. Zhang H, Ding SK, Liu QQ, Ye YY, Xu Q, et al. (2019) Ca2+-Camk-CREB mediates the effect of flavonoids from Scutellaria Baicalensis stems and leaves on the decrease of neuroregeneration induced by composited $\mathrm{A} \beta$ in rats. LJMPNP 5:18-28,

9. Morris R (1984) Developments of a water-maze procedure for studying spatial learning in the rat. J Neurosci Methods 11: 47-60.

10. Xu SY, Bian RL, Chen X (2002) Methodology of pharmacological test. 3 Edition, Beijing: People's Medical Publishing House 2002: 826-830.

11. Bo F (2020) Relationship between the memory behavior of Nupr1 knockout mice C57 and the p-Tau protein in hippocampus by METH exposure. Master Paper, S o u th e r $n$ Medical University 2020.

12. Gong C, Zhang YJ, Zhang M, Shi CY, Liu X, et al. (2020) An open-source multifunctional animal behavior experimental system. Chin J Com Med 30: 92-98.

13. Cantarella G, Di Benedetto G, Puzzo D, Privitera L, Loreto C, et al. (2015) Neutralization of TNFSF10 ameliorates functional outcome in a murine model of Alzheimer's disease. Brain 138: 203-216.

14. Wu XG, Wang SS, Miao H, Cheng JJ, et al. (2016) Scutellaria barbata flavonoids alleviate memory deficits and neuronal injuries induced by composited $A \beta$ in rats. Behav Brain Funct 12: $33-43$.

15. Zheng SP, Xu P (2016) Research of animal behavior assessment about cognitive functions. Chin J Com Med 26: 85-89.

16. Morris RGM, Moser EI, Riedel G (2003) Elements of a neurobiological theory of the hippocampus: the role of activity-dependent synaptic plasticity in memory. Philos Trans R Soc Lond B Biol Sci 358: 773-786.

17. Nunez J (2008) Morris water maze experiment. J Vis Exp19: e897-e897.

18. Zhou JJ, Que JY, Yu WW, Chen ML, Wu ZQ, et al. (2017) Methodology of Morris water maze to detect the level of animal learning and memory. Chin J Ger 37: 6274-6277.

19. Karunakaran S (2020) Unraveling early signs of navigational impairment in APPswe/PS1dE9 mice using Morris water maze. Front Neurosci 14: 568200.

20. Kwon SH, Lee HK, Kim JA, Hong SI, Kim SY, et al. (2011) Neuroprotective effects of Eucommiaulmoides Oliv. Bark on amyloid beta (25-35)-induced learning and memory impairments in mice. Neurosci Lett 487: 123-127.
Copyright: (C2021 Dr. Shang Yazhen. This is an open-access article distributed under the terms of the Creative Commons Attribution License, which permits unrestricted use, distribution, and reproduction in any medium, provided the original author and source are credited. 